\title{
Further Delineation of FKBP14-Related Ehlers-Danlos Syndrome: A Patient with Early Vascular Complications and Non-Progressive Kyphoscoliosis, and Literature Review
}

\section{Chiara Dordoni, ${ }^{1}$ Claudia Ciaccio, ${ }^{1}$ Marina Venturini, ${ }^{2}$ Piergiacomo Calzavara-Pinton, ${ }^{2}$ Marco Ritelli, ${ }^{1}$ and Marina Colombi ${ }^{1 *}$}

\author{
${ }^{1}$ Division of Biology and Genetics, Department of Molecular and Translational Medicine, University of Brescia, School of Medicine, Italy \\ ${ }^{2}$ Division of Dermatology, Department of Clinical and Experimental Sciences, Spedali Civili University Hospital, Brescia, Italy
}

Manuscript Received: 11 December 2015; Manuscript Accepted: 11 April 2016

FKBP14-related Ehlers-Danlos syndrome (EDS) is an extremely rare recessive connective tissue disorder described for the first time in 2012 by Baumann and coworkers. The causal gene, FKBP14, encodes a member of the F506-binding family of peptidyl-prolyl cis-trans isomerases. The paucity of patients described so far makes this disorder poorly defined at clinical level. Here, we report an additional pediatric patient, who is compound heterozygous for a recurrent and a novel FKBP14 mutation, and compare his phenotype with those available in literature. This evaluation confirms that kyphoscoliosis (either progressive or non-progressive), myopathy, joint hypermobility, and congenital hearing loss (sensorineural, conductive, or mixed) are the typical features of the syndrome. Since the patient showed a severe cardiovascular event in childhood and atlantoaxial instability, this report expands the phenotype of the disorder and the allelic repertoire of FKBP14.

() 2016 Wiley Periodicals, Inc.

Key words: Ehlers-Danlos syndrome; FKBP14; vascular complications; joint hypermobility; kyphoscoliosis; atlantoaxial instability

\section{INTRODUCTION}

Ehlers-Danlos syndromes (EDS) are clinically and genetically heterogeneous connective tissue disorders (CTDs) characterized by involvement of skin, joints, ligaments, vasculature, and internal organs [Steinmann et al., 2002]. Six EDS types are included in the Villefranche nosology [Beighton et al., 1998] and several rare variants were more recently delineated [Colombi et al., 2015]. Among these, Baumann et al. [2012] reported a rare recessive variant of EDS characterized by joint hypermobility, progressive kyphoscoliosis, myopathy, and sensorineural hearing loss due to recessive mutations in FKBP14. This gene encodes a member of the F506-binding family of peptidyl-prolyl cis-trans isomerases found in the lumen of the endoplasmic reticulum, where it is thought to
How to Cite this Article:

Dordoni C, Ciaccio C, Venturini M, Calzavara-Pinton P, Ritelli M, Colombi M. 2016. Further delineation of FKBP14-related Ehlers-Danlos syndrome: A patient with early vascular complications and nonprogressive kyphoscoliosis, and literature review.

Am J Med Genet Part A 170A:2031-2038.

catalyze cis-trans-isomerization of peptidyl-prolyl peptide bonds and to accelerate protein folding, particularly of procollagens [Galat, 2003]. To date, eight patients with FKBP14-related EDS from seven independent families have been described: five pediatric ( $\leq 12$ years), one young woman (16 years), and two adults (48 and 42-year-old) [Baumann et al., 2012; Aldeeri et al., 2014; Murray et al., 2014].

Here we report an 8-year-old patient affected by FKBP14-related EDS due to compound heterozygosity for a recurrent and a novel FKBP14 mutation and compare the proband's clinical presentation with the features of the patients available in literature. Our patient presented with vascular rupture at 6 years and showed atlantoaxial instability, never described before in FKBP14-related EDS, thus expanding the clinical phenotype of this rare disorder.

\section{Conflict of interest: none}

*Correspondence to:

Prof. Marina Colombi, Department of Molecular and Translational Medicine, Division of Biology and Genetics, School of Medicine, University of Brescia, Viale Europa 11, 25123 Brescia, Italy.

E-mail: marina.colombi@unibs.it

Article first published online in Wiley Online Library

(wileyonlinelibrary.com): 5 May 2016

DOI 10.1002/ajmg.a.37728 


\section{CLINICAL REPORT}

The patient, an 8-year-old Italian male, was born from healthy nonconsanguineous parents at 36 weeks by cesarean for cephalic presentation. At birth, measurements were within normal range (weight 3 rd-15th centile, length 50th centile). Perinatal presentation was abnormal, with severe muscle hypotonia and poor suck. Newborn hearing screening with Auditory Brainstem Response Audiometry suggested congenital bilateral profound hearing loss. He underwent transtympanic drains due to impaired middle ear ventilation; audiometric testing, performed subsequently, revealed a partial improvement of hearing loss. At age 8 years, he had mixed hearing loss of mild degree. Gross motor milestones were delayed; he walked without help at the age of 2.6 years. Non-progressive and nonsurgical kyphoscoliosis was first observed at 6 months and successfully treated with lumbar orthosis. At the age 2 years, EDS VIA was excluded by lysyl pyridinolines to hydroxylysyl pyridinoline (LP/HP) urinary ratio analysis, which resulted within the normal range. A flexion extension radiograph of the cervical spine performed at the age 6 years revealed an asymptomatic atlantoaxial instability with enlarged atlanto-epistropheal distance in flexion (Fig. 1A, a,b).

Given generalized muscle hypotonia, at age 3 years primary myopathy was suspected and histopathological examination of muscle biopsy specimens revealed changes in muscle fiber diameters, which were first linked to a nemaline myopathy. ACTA1, TPM2, and TPM3 molecular analysis involved in nemaline myopathy types 3, 4, and 1, respectively were negative. Muscle hypotonia slowly improved with psychomotor rehabilitation programs. At age 6 years, the patient was admitted to emergency unit for acute abdomen caused by left hypogastric artery pseudoaneurysm rupture, safely treated with endovascular procedure (Fig. 1A, c). Thoracic and abdominal magnetic resonance angiography (MRA) performed after the vascular event did not show other arterial malformations, such as ectasia, aneurysm, pseudoaneurysm, or tortuosity. Echocardiogram was normal. At age
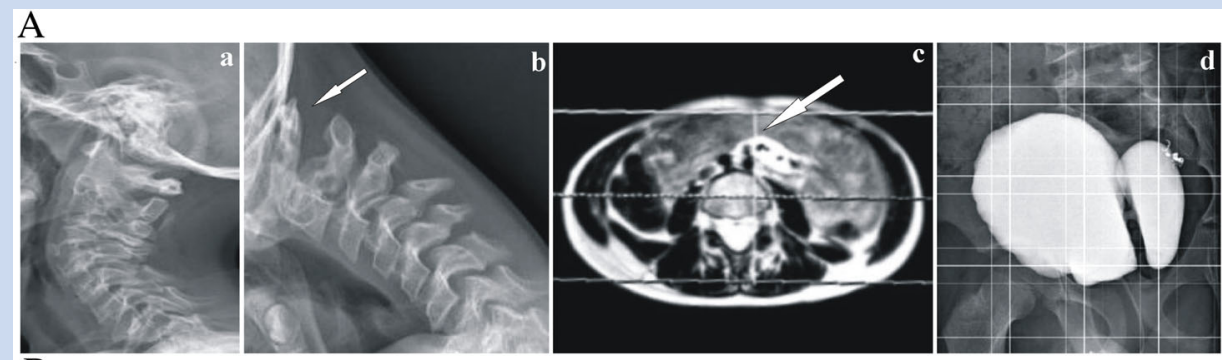

B
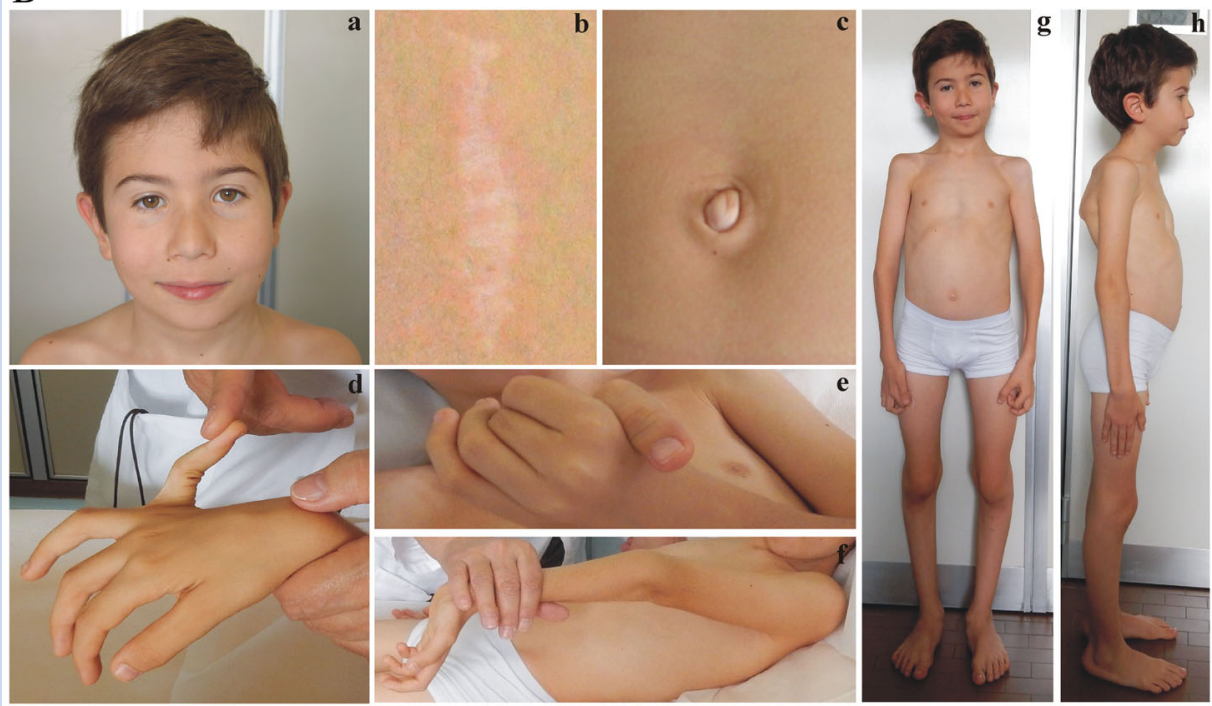

FIG. 1. Radiological and physical findings of the patient. A: Atlanto-axial instability $[a, b]$ with enlarged atlanto-epistropheal distance in flexion [arrow]. Thoraco-abdominal MRA performed after endovascular procedure for left hypogastric artery pseudoaneurysm rupture (arrow] at 7 years [c]; Cystography with contrast at $\mathbf{7}$ years showing a large left postero-lateral bladder diverticulum [d]; B: Epicanthal folds, palpebral skin redundancy, elongated philtrum, micrognathia [a]; enlarged post-bioptic scar [b]; umbilical skin redundancy [c]; hypermobility of the joints of hands and elbow $[d-f]$; generalized muscle hypotonia and atrophy, mild kyphoscoliosis, hyperlordosis, winged scapulae, genua valga, mild pectus excavatum, leg length discrepancy, valgus knee, and pes planus $(g, h$ ]. [Color figure can be seen in the online version of this article, available at http://wileyonlinelibrary.com/journal/ajmga]. 
8 years, an asymptomatic arachnoid cyst of the dorsal spine was successfully surgically removed. Diagnostic evaluation for voiding dysfunction, in particular urinary retention, lead to the diagnosis of a large bladder diverticulum by ultrasound; uroflussometry performed at the age of 8 revealed detrusor dyssynergia of I-II grades (Fig. 1A, d). The patient used braces for ankle instability from 3 to 5 years. At age 6 , vitamin D supplementation was initiated due to radiological findings of osteopenia.

The patient came to our attention at age 8 years; clinical examination revealed normal stature $(1.27 \mathrm{~m}, 25 \mathrm{th}-50$ th centile) and weight $(22 \mathrm{~kg}, 10 \mathrm{th}-25$ th centile), generalized muscle hypotonia with myopathic gait, palpebral and umbilical skin redundancy, epicanthal folds, periorbital creases, light blue sclerae, long philtrum, micrognathia, bifid uvula, an atrophic scar on the knee, an enlarged scar after muscle biopsy, joint hypermobility according to Beighton score (6/9), high palate, winged scapulae, kyphoscoliosis of mild degree, mild pectus excavatum, bilateral valgus elbow, valgus knee, and pes planus (Fig. 1B).

\section{MOLECULAR CHARACTERIZATION}

After informed consent was obtained from the parents, molecular characterization was performed on genomic DNA purified from peripheral blood leukocytes using standard procedures. All exons and intron-flanking regions of FKBP14 were PCR amplified by using optimized genomic primer sets, as previously described [Baumann et al., 2012]. PCR products were purified with ExoSAP-IT (USB Corporation, Cleveland, $\mathrm{OH}$ ) followed by bidirectional sequencing with the BigDye Terminator v1.1 Cycle Sequencing kit on an ABI 3130XL Genetic Analyzer (Applied Biosystems, Carlsbad, CA). FKBP14 sequence analyses revealed compound heterozygosity for the paternal and recurrent c.362dup (p.Glu122Argfs*7) and the maternal novel c.573_576del (p.Glu191del) mutations (Fig. 2), thus confirming the diagnosis of FKBP14-related EDS.

\section{DISCUSSION}

The clinical presentation of the patient reported here was strongly suggestive for FKBP14-related EDS, as he showed all the typical signs of this extremely rare CTD, that is, kyphoscoliosis, muscle hypotonia, and hearing loss. In addition to these cardinal features, at age 6 years, he had a left hypogastric artery dissection, and atlantoaxial instability. Clinical features and biochemical findings of the nine patients affected by FKBP14-related EDS, proven by molecular analysis, are summarized in Tables I and II, respectively. Differential diagnosis should take into account other EDS types with early onset/congenital kyphoscoliosis and myopathic signs, that is, EDS kyphoscoliotic type, CHST14-related EDS, in addition to CTDs with vascular involvement including EDS vascular type, Marfan and Loeys-Dietz syndromes, and myopathies, particularly those with joint laxity, such as Ullrich congenital dystrophy and Bethlem myopathy. The hearing loss distinguishes FKBP14-related EDS from these disorders. Moreover, FKBP14-related EDS differs from EDS kyphoscoliotic type by the normal ratio of urinary LP/HP crosslinks [Rohrbach et al., 2011], from CHST14-related EDS by the absence of congenital contracture of thumbs and

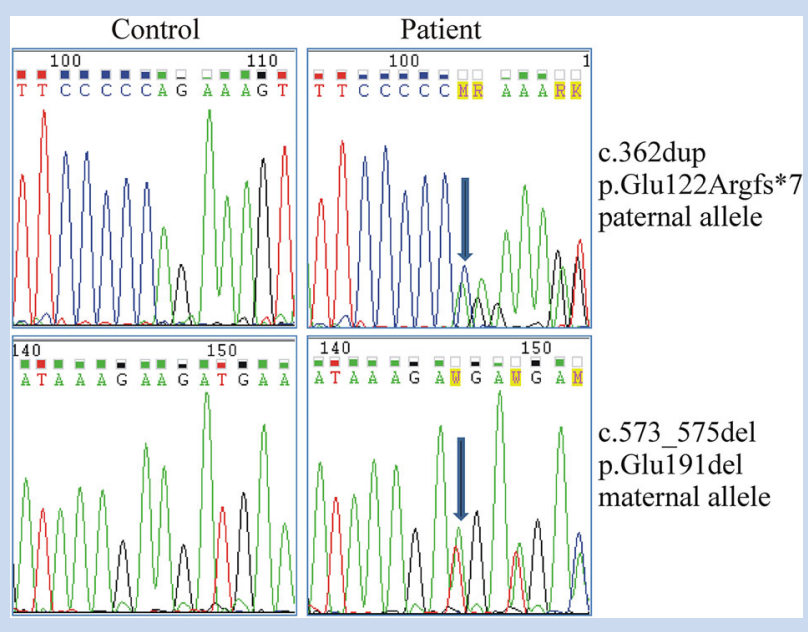

FIG. 2. Sequence chromatograms showing the positions of the recurrent and paternal inherited c.362dup (p.Glu122Argfs* 7 ) and the novel maternal c.573_575del (p.Glu191del) mutations that were detected in the proband in compound heterozygosity in exon 3 and exon 4 of FKBP14, respectively. Mutations are annotated according to HGVS nomenclature (http://www.hgvs. org/mutnomen; seq. refs: NM_017946.3; NP_060416.1]. [Color figure can be seen in the online version of this article, available at http://wileyonlinelibrary.com/journal/ajmga].

fingers, and from the above mentioned CTDs with vascular involvement by the absence of a typical facial appearance and visceral rupture, marfanoid habitus, craniosynostosis, and widespread aortic/arterial aneurysms [Colombi et al., 2015]. Absence of respiratory muscle failure, improvement of muscle hypotonia with age and presence of an unspecific myopathic pattern on electrophysiological and muscle biopsy studies are useful aids to differentiate FKBP14-related EDS form primary myopathies [Baumann et al., 2012].

All the reported FKBP14-related EDS patients showed the cardinal features of the disease, that is, hearing loss, early-onset kyphoscoliosis, and myopathic signs, though with high variability (Table I). Hearing impairment can vary from sensorineural (6/8), to conductive (1/8), or mixed (present case) (Table I). In our patient, because of the mixed origin of this sign, hearing improved after transtympanic drains. Kyphoscoliosis is noted at a mean of 12 months (range 2-18 months) and it can be non-progressive (three patients including ours) or progressive (6). Orthotic treatment seemed successful in case of non-progressive kyphoscoliosis, as in our patient; progressive kyphoscoliosis required a surgical approach. Myopathic signs include muscle hypotonia and atrophy (9/9), poor head control in infancy (7/7), and delayed motor development (9/9) (Table I). Muscular weakness seems to regress with age and all of the subjects-but one-became able to walk at the mean age of 33 months. The outcome is very variable and the final ability to walk ranges from myopathic gait, impossibility of walking without aids, to a motor self-sufficiency from $200 \mathrm{~m}$ to $1 \mathrm{~km}$ (Table I). Muscle biopsy always shows pathological results with a broad spectrum of findings; creatine kinase generally results 


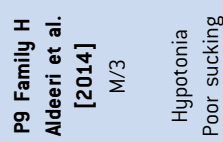

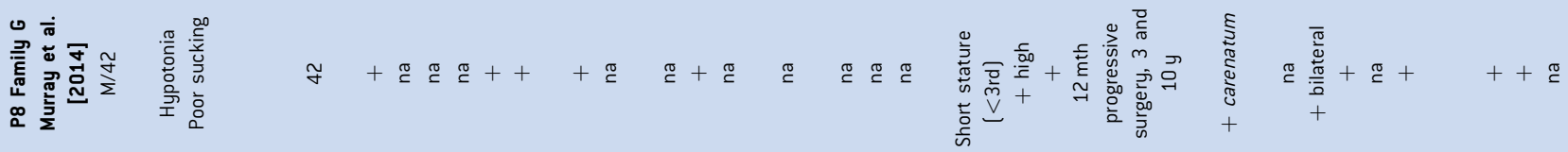

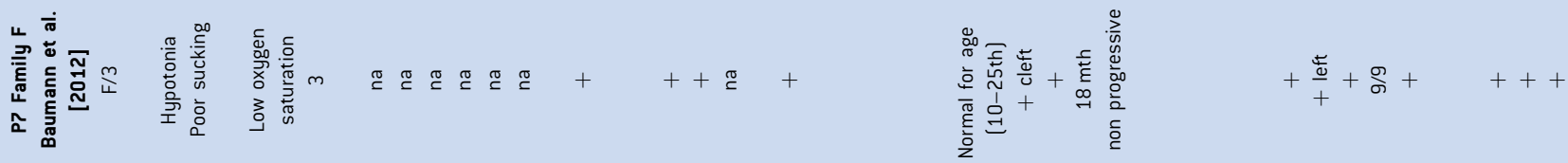

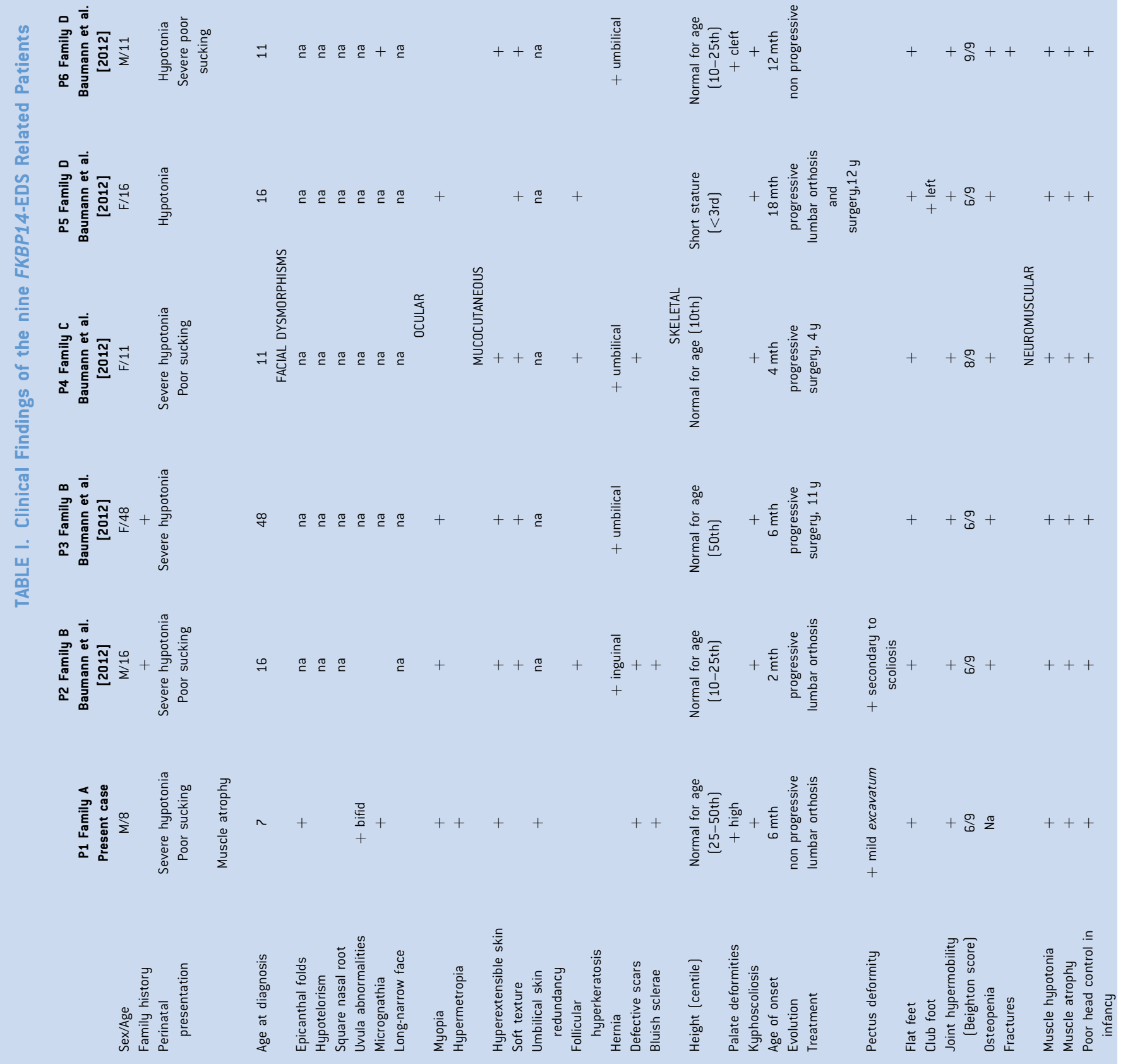




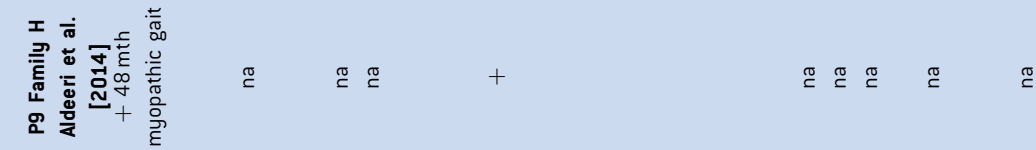

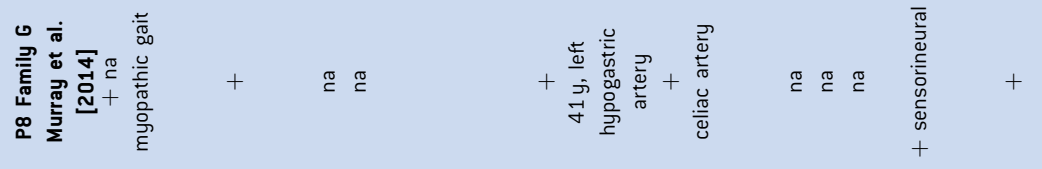

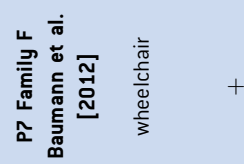

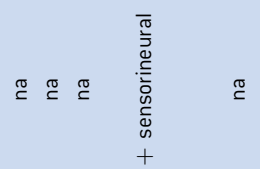

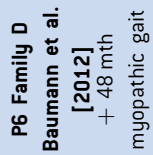

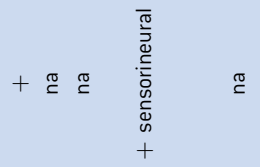

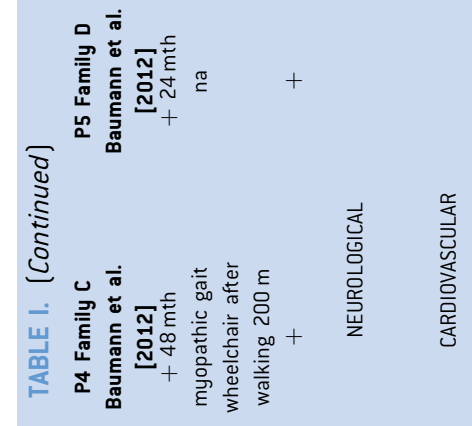

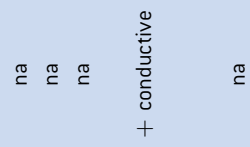

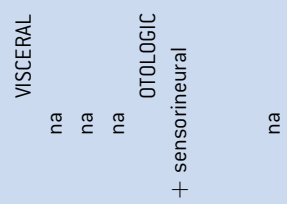

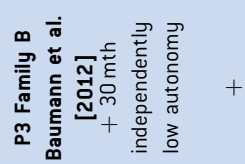

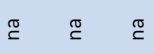

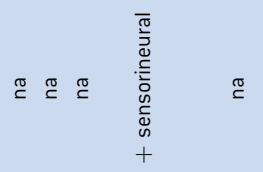

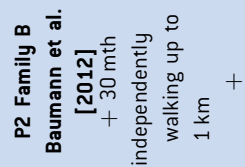

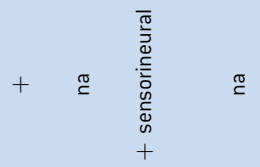

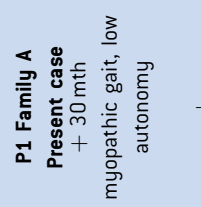

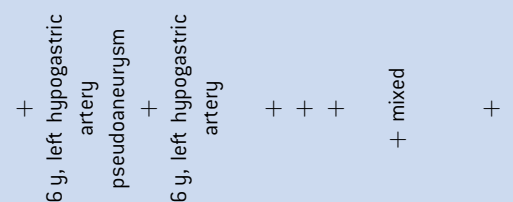

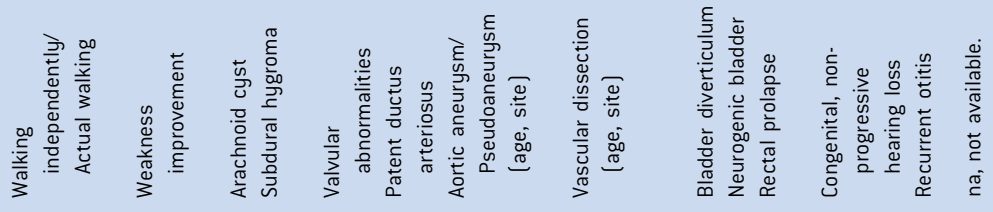




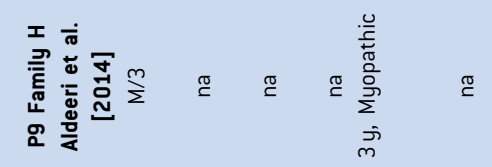

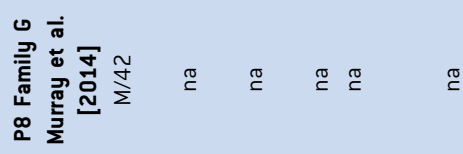

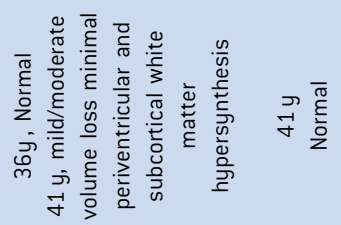

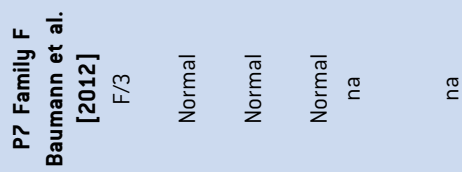

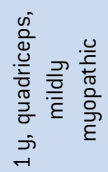

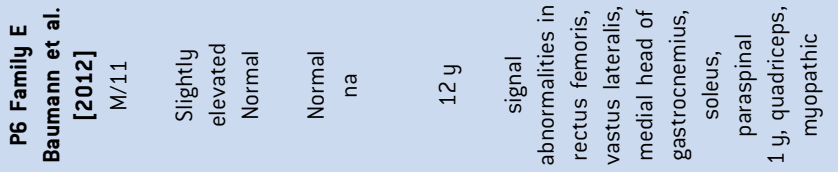

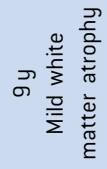

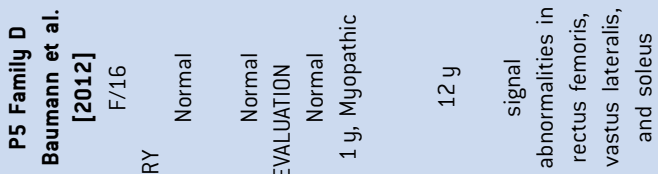

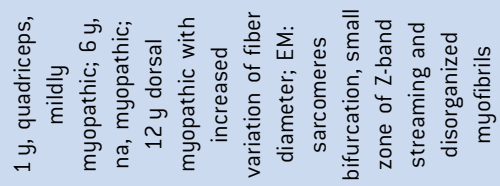

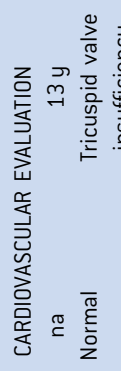

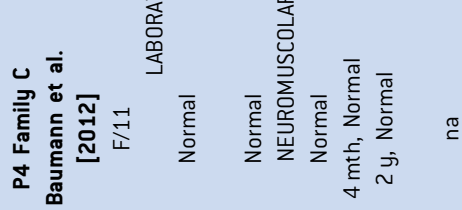

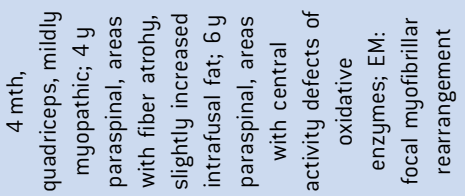

$\stackrel{\Xi}{\simeq}$

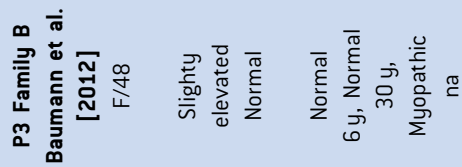

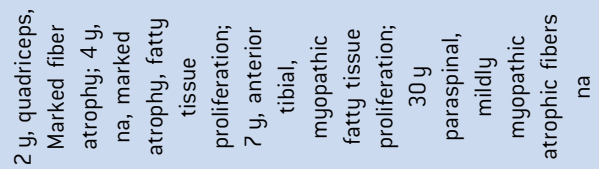

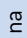

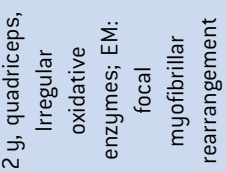

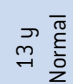

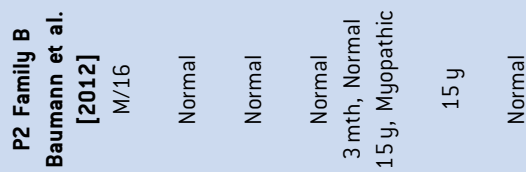

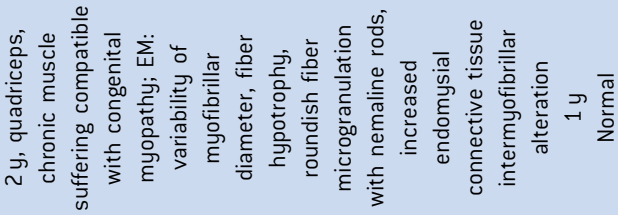

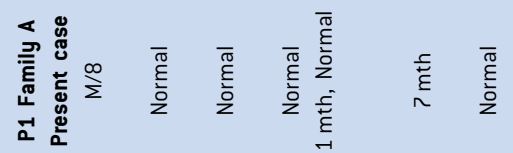

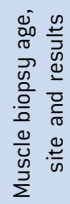

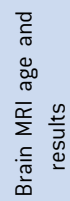



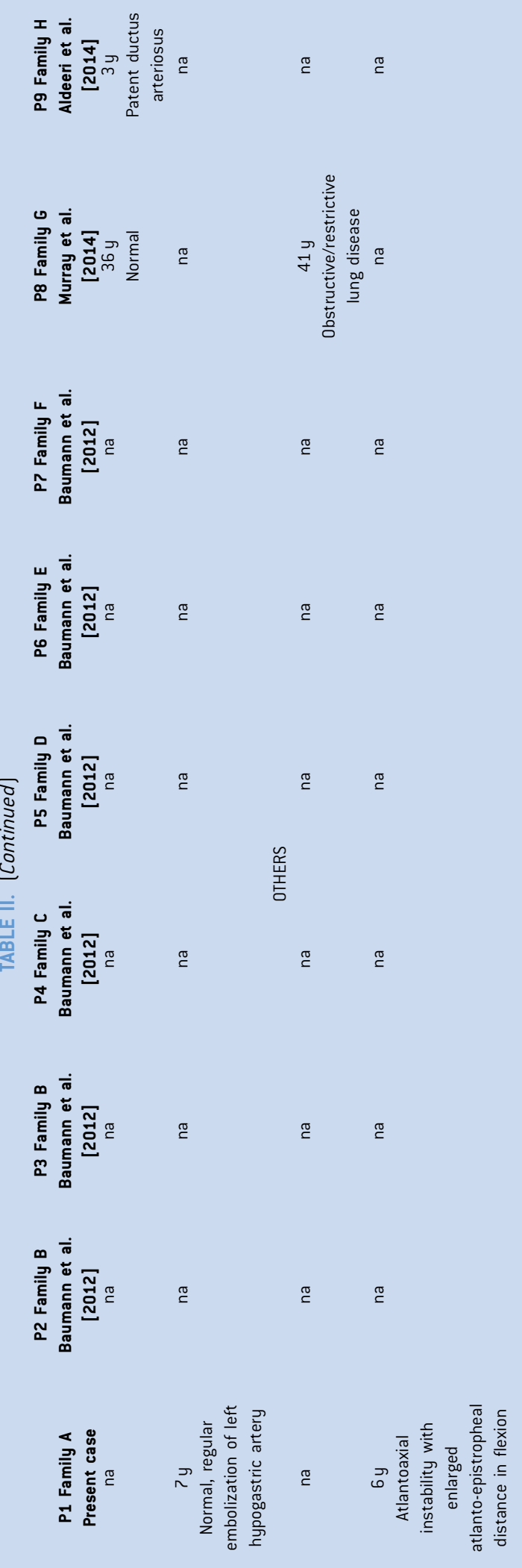

\begin{tabular}{|c|c|c|c|c|}
\hline $\begin{array}{l}\frac{\overline{0}}{0} \\
\frac{0}{0} \\
\frac{0}{0} \\
\text { ज } \\
\frac{0}{0} \\
\frac{0}{0}\end{array}$ & 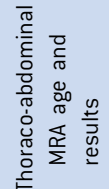 & 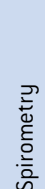 & 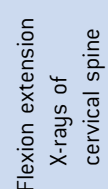 & $\begin{array}{l}\frac{0}{0} \\
\frac{\pi}{\pi} \\
\frac{\pi}{\pi} \\
\frac{\pi}{\pi} \\
\frac{0}{c} \\
\frac{0}{c}\end{array}$ \\
\hline
\end{tabular}

within the normal range (5/7) and electromyographic pattern can vary with age (Table II).

In addition to these hallmarks, $F K B P$-14-related EDS patients show a variable constellation of signs due to connective tissue impairment affecting ocular, mucocutaneous, skeletal, nervous central, and cardiovascular systems (Table I). The cardiovascular risk in FKBP14-related EDS is not well defined (Table I). Vascular complications are described in P8 (Table I), who presented a celiac artery pseudoaneurysm rupture at the age of 41 , and in the older sister of P3 (Table I), likely affected but without molecular confirmation, who died due to unspecified aortic rupture at age 12 years. Celiac artery pseudoaneurysm rupture was observed in our patient at age 6 (Fig. 1). Atlantoaxial instability is reported here for the first time, the other patients presented uncomplicated joint hypermobility without recurrent dislocations/sprains or chronic pain (mean value of Beighton score 7/9) (Table I). Facial dysmorphisms are not always described and a facial "gestalt" is not recognizable, some patients had epicanthal folds (3/3), micrognathia (3/4), hypotelorism (1), square nasal root (1), or long-narrow face (1) (Table I). Height is generally within the normal range, but at lower level (10th-25th centile in 4 of 8 ), two patients had short stature (lower than third centile) (Table I). Tissue fragility in FKBP14-related EDS may lead to large bladder diverticulum (Fig. 1) or rectal prolapse (present case).

In conclusion, our report supports many of the clinical data available in literature for FKBP14-related EDS, confirming the variability observed for the cardinal features. In addition, this report describes a potentially life-threatening vascular complication in early pediatric age and atlantoaxial instability, suggesting the need for FKBP14-related EDS patients of tailored follow-up that includes cardiovascular monitoring, that is, cerebral, thoracic and abdominal MRA, and cervical dynamic radiograph. Further studies are needed to better delineate the phenotype of FKBP14related EDS.

\section{ACKNOWLEDGMENTS}

The authors thank the patient's parents for their cooperation during the diagnostic process of their son.

\section{REFERENCES}

Aldeeri AA, Alazami AM, Hijazi H, Alzahrani F, Alkuraya FS. 2014. Excessive redundant umbilical skin as a potential early clinical feature of Morquio Syndrome and FKBP14-related Ehlers-Danlos syndrome. Clin Genet 86:469-472.

Baumann M, Giunta C, Krabichler B, Rüschendorf F, Zoppi N, Colombi M, Bittner RE, Quijano-Roy S, Muntoni F, Cirak S, Schreiber G, Zou Y, Hu Y, Romero NB, Carlier RY, Amberger A, Deutschmann A, Straub V, Rohrbach M, Steinmann B, Rostásy K, Karall D, Bönnemann CG, Zschocke J, Fauth C. 2012. Mutations in FKBP14 cause a variant of Ehlers-Danlos syndrome with progressive kyphoscoliosis, myopathy, and hearing loss. Am J Hum Genet 90:201-216.

Beighton P, De Paepe A, Steinmann B, Tsipouras P, Wenstrup RJ. 1998. Ehlers-Danlos syndromes: Revised nosology, villefranche. Am J Med Genet 77:31-37.

Colombi M, Dordoni C, Chiarelli N, Ritelli M. 2015. Differential diagnosis and diagnostic flow chart of joint hypermobility 
syndrome/Ehlers-Danlos syndrome hypermobility type compared to other heritable connective tissue disorders. Am J Med Genet C Semin Med Genet 169C:6-22.

Galat A. 2003. Peptidylprolyl cis/trans isomerases (immunophilins): Biological diversity-targets-functions. Curr Top Med Chem 3:1315-1347.

Murray ML, Yang M, Fauth C, Byers PH. 2014. FKBP14-related EhlersDanlos syndrome: Expansion of the phenotype to include vascular complication. Am J Hum Genet 164:1750-1755.
Rohrbach M, Vandersteen A, Yiş U, Serdaroglu G, Ataman E, Chopra M, Garcia S, Jones K, Kariminejad A, Kraenzlin M, Marcelis C, Baumgartner M, Giunta C. 2011. Phenotypic variability of the kyphoscoliotic type of Ehlers-Danlos syndrome (EDS VIA): Clinical, molecular, and biochemical delineation. Orphanet J Rare Dis 23:6-46.

Steinmann B, Royce PM, Superti-Furga A. 2002. The Ehlers-Danlos syndrome. In: Royce PM, Steinmann B, editors. Connection tissue and its heritable disorders, 2nd edition. New York: Wyley-Liss. pp 431-523. 